\title{
Psychogenic Blindness: A Rare Presentation
}

\author{
Dutta A', Poudel R ${ }^{2}$, Thapa LJ $^{3}$, Pokhrel B ${ }^{4}$ \\ 1.Lecturer, Manmohan Memorial Medical College, Kathmandu 2. Consultant Neurologist, The Grande \\ International Hospital, Kathmandu 3. Consultant Neurologist, COMS, Bharatpur, chitwam 4. Resident,COMS, \\ Bharatpur, Chitwan
}

\begin{abstract}
:
Psychogenic blindness is a type of dissociative sensory loss which is characterized by unilateral or bilateral loss of vision/poor vision in the absence of any organic cause. we present a case of psychogenic blindness in a 21 years old female, 32 weeks primigravida who had presented with both eye complete loss of vision of 6 days duration. On admission, ophthalmological and neurological examination revealed no abnormality of visual pathways. Psychoeducation and reaasurance lead to complete visual recovery after $12 \mathrm{hrs}$ of first psychiatric intervention.
\end{abstract}

Keywords: Dissociation, Blindness, Non organic

\section{INTRODUCTION}

Dissociative disorders are disorders in which there is disruption in the usually integrated functions of memory, identity, or perception of the environment. ${ }^{1}$ In the diagnostic classification system of ICD-10(DCR) this category falls under the broad group of Neurotic, Stress related, and Somatoform disorders. DSM-IV adopts a different approach - conversion disorders are classified under 'somatoform disorders'.

Visual complaints without evidence of contributory ocular or non-ocular pathology are not infrequent presentations to the Emergency department, and are said to account for approximately $1 \%$ of visual problems seen by the ophthalmologist. ${ }^{2}$

The wide spectrum of terminology used to describe visual disturbance without a physical basis, which includes 'functional', 'hysterical', 'supratentorial', and 'psychosomatic', has resulted in considerable confusion .

Psychogenic blindness is more common among younger age-group and females. ${ }^{2}$ It may present as blurring of vision or difficulty in reading. ${ }^{3}$ However cases of psychogenic blindness that convincingly mimic true blindness are rare because people lack an intuitive knowledge of visual pathways. ${ }^{3}$
Psychogenic blindness occurs in malingering and various psychiatric conditions. ${ }^{3}$ The most frequently reported non-organic visual complaints presenting to ophthalmologists include a reduction of visual acuity with or without loss of field (36-80\%). ${ }^{4}$

The pathogenesis of psychogenic visual complaints remains obscure. Conversion and somatization disorders, as well as hypochondriasis, have all been implicated in the process . A conversion reaction uses a physical symptom to express a psychological conflict.

Disabling hysterical blindness presents more difficulties. A diagnosis of non-organic visual loss is made when the visual acuity is shown to be better than subjectively alleged, and where the functional integrity of the afferent visual pathway is confirmed by clinical examination with or without supplementary electrophysiological investigations.

Findings not consistent with an organic aetiology under such circumstances include a smooth entrance into the consulting room, an intact menace reflex, flinching with increased illumination or a failure to direct the eyes towards their own hands during manual tasks. ${ }^{5}$ To expose intact vision, spinning a vertically striped surface will elicit a optokinetic nystagmus unless true blindness is present. likewise having patients stare at a large moving mirror irresistibly compels them to follow their own image. 
Various tests which have been recommended in ophthalmological practice for the diagnosis of psychogenic blindness are Fogging test, Prism shift test, Polarizing lens test, Stereoscopic test, Preferential looking. ${ }^{5}$

Tubular or spiral visual fields may be found on testing by perimetry. ${ }^{6}$ Evoked-potential studies help to demonstrate intact visual pathways. If clinical bedside tests are inconclusive, EEG may help. Alpha rhythm overlying the occipital lobes of patients at rest with their eyes closed, and loss of that rhythm when they open their eyes, indicates an intact visual system. ${ }^{3}$

Treatment methods used in the past included psychoanalysis, sensory deprivation, confrontation, and placebo treatments. However, the cornerstone of management for psychogenic or feigned visual complaints remains reassurance by a concerned physician that there is no brain or eye disease. ${ }^{7}$ The natural history of non-organic visual loss should be explained and the expectation of complete visual recovery should be expressed. Use of placebo treatments such as eye drops, orthoptic exercises or spectacles are not recommended as they will only serve to undermine the reassurance. ${ }^{7}$ To the contrary some authors believe that offering an eyeglass with lenses having negligible optical strength allows patients to extract themselves from psychogenic blindness without embarrassment. ${ }^{8}$

Terms such as 'pretending' should be avoided, and punitive measures should be actively discouraged. The possibility that the symptoms may be a somatic expression of stress should also be explained, and that improved communication between family members may accelerate the visual recovery.

\section{PROGNOSIS}

Using the therapeutic approach described above, between $45 \%$ and $78 \%$ experience complete resolution of all visual symptoms. ${ }^{4}$ Good prognostic indicators include young age and absence of any associated psychiatric disease. ${ }^{4}$

\section{CASE REPORT}

21 yrs old married, hindu female from middle socioeconomic status, educated upto bachelors in management, primigravida at 36 wks of pregnancy (planned pregnancy, wanted child, preference for a daughter though gender of child not known), presented with sudden, painless loss of vision of 6 days duration. History revealed a spontaneous threatened pregnancy one and half months ago, followed by a fall in the bathroom - leading to worries about viability of foetus, associated with low mood, anhedonia, apprehension. There was also worries about rejection by in laws following such incidence. 5 days prior to hospital admission, following a panic attack patient complained of not being able to see anything. During the stage of blindness, however patient could make out bright light, the source of light, but couldn't clearly see objects or people in her vicinity. There was no headache or vomiting associated, appetite was normal but sleep decreased. On assessment there was no history of intrafamilial conflicts, patient was staying with her husband, sharing good interpersonal relationship. There was no history of any bodily injury. There were no complains of diplopia, tunnel vision, hemianopia. Neither was there any history of hypertension or diabetes mellitus.

On examination, General condition was fair with stable vitals and Systemic Examination revealed no abnormality except 36weeks pregnancy, Optokinetic nystagmus, and Positive Menace test. All routine blood and urine tests were within normal range. Mental State examination revealed Anxious affect and worrisome thoughts.

Diagnosis of Psychogenic Blindness was made and was revealed to patient and family members, and explained about the psychological origin of the disease. Secondary gain was cut down, reassurance was provided to patient. Education about the stages of labour and the normal outcome of pregnancy was provided reinforced by the fact that medically her foetus was normal and a favourable outcome was expected. After few hrs of this, vision improved and by next morning- $90 \%$ return of normal vision.

\section{DISCUSSION}

The above mentioned case represents a disruption in the integration of visual modality of perception with the environment. As discussed by Merskey H, the most representative examples of this type of symptom include claims of blindness, deafness, paralysis, loss of speech, and certain types of memory disturbance, including fugue states. ${ }^{6}$ 
In most of the related literatures the term Non Organic Visual Loss (NOVL) has been used to describe any visual disturbance where there is no detectable dysfunction of structures between cornea and occipital cortex. The literature indicates that a motor conversion disorder has a high incidence and the visual conversion disorder a lower incidence. Exact figures are lacking. ${ }^{8}$

Of 42 patients with psychogenic or feigned visual complaints assessed by a psychiatrist in the series reported by Kathol et al, 22 (52\%) were found to have psychiatric syndromes. ${ }^{9}$

In the above mentioned case a comorbid moderate depressive episode with panic attacks were found, which definitely required psychiatric intervention for symptom relief. However the findings of one case cannot be generalized to all cases of NOVL/Psychogenic blindness. Nevertheless since there is an association of intrapsychic conflict in the formation of conversion symptoms, theoretically psychiatric intervention may be required in all cases of conversion disorder henceforth in psychogenic blindness.

\section{REFERENCES:}

1. Sadock BJ, Sadock VA. Synopsis of Psychiatry. 10 th ed. Philadelphia: Lippincott Williams and Wilkins; 2007. Chapter 20, Dissociative Disorders; P.665-679.

2. Kauffmann DM, Milstein MJ . Neurology for psychiatrists. $7^{\text {th }}$ ed: Elsevier;2013. Chapter 12, Visual Disturbances; $p$ ?

3. Kathol RG, Cox TA, Corbett JJ, Thompson HS. Functional visual loss. Follow-up of 42 cases. Arch Ophthalmol 1983;101:729-35.

4. Merskey H. Conversion and Dissociation. In Gelder GM, Lopez-lbor JJ, Andreason N, editors. New Oxford Textbook of Psychiatry. First edition. Oxford. Oxford University Press; 2003.

5. Beatty S. NOVL. Postgraduate medical journal 1999;75:201-207.

6. Brandenburg S, Hendriks A. Psychogenic Blindness: A Strategic Model in Two Traces. Paper presented at $7^{\text {th }}$ General Assenmbly ICEVI- European Conference in Dublin; July 2009.

7. Kathol RG, Cox TA, Corbett JJ, Thompson HS, Clancy J. Functional visual loss: I A true psychiatric disorder? Psychol Med 1983;13:307-14.

8. Sletteberg $O$, Bertelsen $T$, Hovding $G$. The prognosis of patients with hysterical visual impairment. Acta Ophthalmol 1989;67:159-63.

9. Kathol, R.G., Cox, T.A., Corbett, J.J., Thompson, H.S., and Clancy, J. (1983). Functional visual loss. I: A true psychiatric disorder? Psychological Medicine, 13, 307-14. 